\title{
3D Intraventricular Flow Mapping from Colour Doppler Images and Wall Motion
}

\author{
Alberto Gómez ${ }^{1, \star}$, Adelaide de Vecchi ${ }^{1}$, Kuberan Pushparajah ${ }^{1,2}$, \\ John Simpson ${ }^{1,2}$, Daniel Giese ${ }^{1}$, Tobias Schaeffter ${ }^{1}$, and Graeme Penney ${ }^{1}$ \\ ${ }^{1}$ King's College London, Division of Imaging Sciences, London, UK \\ ${ }^{2}$ NHS Trust, Guy and St Thomas' Hospital, London, UK
}

\begin{abstract}
We propose a new method to recover 3D time-resolved velocity vectors within the left ventricle (LV) using a combination of multiple registered 3D colour Doppler images and LV wall motion. Incorporation of wall motion, calculated from 3D B-Mode images, and the use of a multi-scale reconstruction framework allow recovery of 3D velocity over the entire ventricle, even in regions where there is little or no Doppler data.

Our method is tested on the LV of a paediatric patient and is compared to 2D and 3D flow Magnetic Resonance Imaging (MRI). Use of wall motion information increased stroke volume accuracy by $14 \%$, and enabled full 3D velocity mapping within the ventricle. Velocity distribution showed good agreement with respect to MRI and vortex formation during diastole was successfully reconstructed.
\end{abstract}

Keywords: Colour Doppler Echocardiography, 3D Velocity Reconstruction, Flow Patterns, Cardiac Motion, Multiple Views, and Registration.

\section{Introduction}

Accurate $3 \mathrm{D}$ characterisation of intracardiac flow has the potential to allow new metrics to be used for cardiac assessment, such as cardiac efficiency and viscous losses [1, and to improve diagnosis of many cardiac conditions which are characterised by abnormal direction and velocity of blood flow.

Latest advances in temporally resolved 3 directional flow-encoded 3D MRI (4D flow MRI) over the past years have enabled non-invasive measurements of $3 \mathrm{D}$ velocities within the cardiovascular system. However, MRI is more expensive than echocardiography (echo) and requires long acquisition times. Furthermore, MRI is incompatible with a number of cardiac implants and is used on selected patients always after an echo exam.

\footnotetext{
* This research was part funded by the EPSRC grant Grand Challenges and by the EPSRC grant Intelligent Imaging: Motion, Form and Function Across Scale. This research was also supported by the National Institute for Health Research (NIHR) Biomedical Research Centre at Guy's and St Thomas' NHS Foundation Trust and King's College London. The views expressed are those of the authors and not necessarily those of the NHS, the NIHR or the Department of Health.
} 
The introduction of 2D matrix array echo technology allows rapid acquisition of 3D B-Mode and 3D colour Doppler volumes. Nevertheless, Doppler velocity information remains a $1 \mathrm{D}$ projection of the true $3 \mathrm{D}$ velocity vector along the echo beam direction. Some authors have proposed to combine wall motion with $2 \mathrm{D}$ colour Doppler images to reconstruct 2D intraventricular velocity [2], but extension to 3D has not been achieved so far because the continuity equation in 3D is not linearly separable. Others have proposed crossed-beam techniques to recover 2D [34] and 3D [5] blood velocity. One limitation of crossed-beam techniques is that $3 \mathrm{D}$ velocity can only be reconstructed where at least 3 input colour Doppler images overlap. However, achieving full coverage of the ventricular cavity with at least 3 different 3D input views is difficult because acoustic access is limited by anatomy and because the computational requirements of colour Doppler images results in a significantly reduced Field of View (FoV) compared to B-Mode images. For example, the blue region in Fig. 1, which shows the region in which 3 or more colour Doppler views overlap, does not cover the whole of the LV. As a result, a crossed-beam approach would not be able to reconstruct $3 \mathrm{D}$ velocity over the entire ventricle.

This paper introduces two main novelties over previous crossed-beam techniques: 1) the incorporation of wall motion to achieve full ventricular velocity mapping by imposing free-slip condition at the LV wall, and 2) the multi-scale implementation of the reconstruction method. The proposed method is applied to a paediatric patient. Quantitative validation is carried out by comparison with the gold standard for non-invasive flow measurement, 2D flow MRI. For qualitative validation, flow patterns and $3 \mathrm{D}$ velocity profiles were compared to 3D flow MRI measurements.

\section{Methods}

The proposed method reconstructs $3 \mathrm{D}$ velocity in 3 steps:

1. Echo images are registered to each other using an intensity-based similarity metric based on phase congruency between multi-view B-Mode images [6].

2. Wall motion is estimated by non-rigid registration of consecutive frames in a B-Mode sequence (Sec. 2.1).

3. Registered colour Doppler images and wall motion are incorporated into a B-spline based reconstruction algorithm (Sec. 2.2).

The $3 \mathrm{D}$ velocity field is calculated with a multi-scale approach (Sec. 2.3) which provides means to analyse coarse and fine scale flow structures.

\subsection{Wall Motion Estimation and Incorporation}

Wall motion was estimated using non-rigid registration on every two consecutive frames of a standard apical B-Mode sequence, where the whole LV was visible (Fig. 1). Registrations were carried out with IRTK [7, using cross-correlation as a similarity measure and a B-Spline grid of isotropic spacing set to $10 \mathrm{~mm}$. 
A manual ventricular segmentation was carried out for the first frame, which was then propagated to all the other frames in the sequence using the non-rigid registration motion fields as shown in Fig. 1 (a). Figure 11 (b) illustrates how wall motion relates to blood velocity, so that one component of the velocity (orthogonal to the LV wall) can be inferred from wall motion and incorporated into the reconstruction problem together with Doppler data.
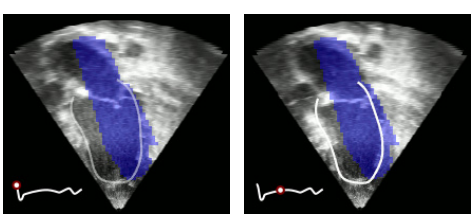

(a)

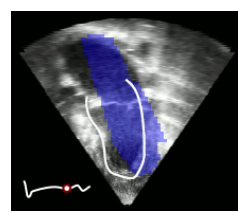

rœ

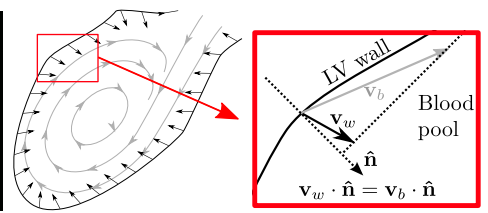

(b)

Fig. 1. Wall motion estimation and incorporation. (a) Overlap of input views and wall motion detection. The region where 3 or more colour Doppler views overlapped is represented with a blue overlay. Wall motion was calculated by registering each pair of consecutive frames. The first frame (left) was manually segmented (LV wall is shown in white) and the segmentation was propagated through the rest of the frames with the calculated motion fields. (b) A free-slip condition is imposed by assuming that the component of blood velocity orthogonal to the $\mathrm{LV}$ wall $\left(\mathbf{v}_{\mathbf{b}} \cdot \hat{\mathbf{n}}\right)$ at the $\mathrm{LV}$ wall is equal to the component of the wall motion orthogonal to the wall $\left(\mathbf{v}_{\mathbf{w}} \cdot \hat{\mathbf{n}}\right)$.

\subsection{D Velocity Reconstruction}

The general framework for 3D velocity reconstruction from multiple views using a B-spline grid is described in [5. In this paper, this method is extended to incorporate wall motion at multiple scales as follows. Input data for the reconstruction algorithm was a list of 3 -tuples $\{m, \hat{\mathbf{d}}, \mathbf{p}\}$, where $m$ was the velocity value along direction $\hat{\mathbf{d}}$ at position $\mathbf{p}=\left[\begin{array}{lll}p_{x} & p_{y} & p_{z}\end{array}\right]^{\top}$. For each input $3 \mathrm{D}$ colour Doppler image, $m$ was the Doppler velocity value, $\hat{\mathbf{d}}$ was the ultrasound beam direction and $\mathbf{p}$ was the coordinates of the voxel centre. For the wall motion, each $\{m\}_{r}$ was equal to $\mathbf{V}_{\mathbf{w}} \cdot \hat{\mathbf{n}}$, $\hat{\mathbf{d}}$ was the normal to the wall, and $\mathbf{p}$ was the coordinates of the node of the surface mesh.

The three components of the velocity were recovered by fitting a smooth vector field $\mathbf{V}$ to the input data (list of 3-tuples). The smooth vector field was expressed in a B-spline basis:

$$
v_{\gamma}(\mathbf{p})=\sum_{i=1}^{N_{g}^{i}} \sum_{j=1}^{N_{g}^{j}} \sum_{k=1}^{N_{g}^{k}} c_{i, j, k}^{\gamma} \beta_{s, i}^{n}\left(p_{x}\right) \beta_{s, j}^{n}\left(p_{y}\right) \beta_{s, k}^{n}\left(p_{z}\right) \quad \gamma \in\{x, y, z\}
$$

where $\beta_{s, l}^{n}(p)=\beta^{n}(p / s-l)$ is the B-spline basis function of degree $n$, the B-spline grid has a size of $N_{g}^{i} \times N_{g}^{j} \times N_{g}^{k}$ and $\left\{c^{x}, c^{y}, c^{z}\right\}_{i, j, k}$ are the B-spline coefficients. The parameter $s$ is the distance between grid nodes and determines the resolution 
of the resulting vector field (i.e. its scale). Equation (11) can be rewritten in matrix form as $\mathbf{V}=\mathbf{S C}$, where $\mathbf{V}$ and $\mathbf{C}$ are column vectors which contain the three components of the velocity and the B-spline coefficients respectively. $\mathbf{S}$ is the B-spline sampling matrix defined by

$$
\begin{gathered}
\mathbf{S}=\left[\begin{array}{ccc}
\mathbf{S}_{\mathbf{s}} & 0 & 0 \\
0 & \mathbf{S}_{\mathbf{s}} & 0 \\
0 & 0 & \mathbf{S}_{\mathbf{s}}
\end{array}\right] \\
\left\{\mathbf{S}_{\mathbf{s}}\right\}_{r,\left((i-1) N_{g}^{j}+j-1\right) N_{g}^{k}+k}=\beta_{s, i}^{n}\left(p_{r, x}\right) \beta_{s, j}^{n}\left(p_{r, y}\right) \beta_{s, k}^{n}\left(p_{r, z}\right)
\end{gathered}
$$

The reconstruction error $J_{\text {rec }}(\mathbf{c})$ was defined as the sum of the point-wise squared difference between the measured values $m$ and the projection of the reconstruction velocity $\boldsymbol{v}$ along the sampling direction $\hat{\mathbf{d}}$ :

$$
J_{r e c}(\mathbf{c})=\sum_{\mathbf{p}}\|\hat{\mathbf{d}}(\mathbf{p}) \cdot \mathbf{v}(\mathbf{p})-m(\mathbf{p})\|^{2}=(\mathbf{D S C}-\mathbf{m})^{\top}(\mathbf{D S C}-\mathbf{m})
$$

where $\mathbf{m}=\left[m_{1} \ldots m_{K}\right]^{\top}, \mathbf{D}=\left[\begin{array}{lll}\mathbf{D}_{\mathbf{x}} & \mathbf{D}_{\mathbf{y}} & \mathbf{D}_{\mathbf{z}}\end{array}\right]$ and $\mathbf{D}_{\gamma}$ is a diagonal matrix with the $\gamma=\{x, y, z\}$ component of $\hat{\mathbf{d}}$ in the diagonal. The coefficients $\mathbf{C}$ that minimise the error (3) in the Least Mean Squares (LMS) sense can be found by $\frac{\partial}{\partial \mathbf{C}} J_{\text {rec }}(\mathbf{C})=0$.

The problem can be further regularised by penalising the divergence of the reconstructed velocity field since blood flow is incompressible. This adds a divergence term to (3) and yields a new error function to be minimised:

$$
J(\mathbf{c})=(1-\lambda) J_{r e c}(\mathbf{c})+\lambda \sum\|\nabla \cdot \mathbf{v}\|^{2}=(1-\lambda) J(\mathbf{C})+\lambda \dot{\mathbf{S}}^{\top} \dot{\mathbf{S}}
$$

where $\lambda$ is a weighting factor which controls the amount of regularisation, $\dot{\mathbf{S}}=$

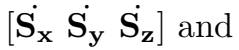

$$
\left\{\dot{\mathbf{S}}_{x}\right\}_{r,\left((i-1) N_{g}^{j}+j-1\right) N_{g}^{k}+k}=\dot{\beta}_{a, i}^{n}\left(p_{x}^{r}\right) \beta_{a, j}^{n}\left(p_{y}^{r}\right) \beta_{a, k}^{n}\left(p_{z}^{r}\right)
$$

with equivalent equations for $\dot{\mathbf{S}}_{y}$ and $\dot{\mathbf{S}}_{z}$. The solution coefficients are $\mathbf{C}=((1-$ त) $\left.A_{r e c}+\lambda A_{d i v}\right)^{-1} \mathbf{b}$ where $\mathbf{A}_{r e c}=\mathbf{S}^{\top} \mathbf{D}^{\top} \mathbf{D S}, A_{d i v}=\dot{\mathbf{S}}^{\top} \dot{\mathbf{S}}$ and $\mathbf{b}=\mathbf{S}^{\top} \mathbf{D}^{\top} \mathbf{m}$. The solution velocity at the points sampled by $\mathbf{S}$ can be obtained as $\mathbf{V}=\mathbf{S C}$.

\subsection{Multi-scale Approach}

Motivation for the Multi-scale Approach. Data fitting with a B-spline grid can be unstable if data points are sparse compared to the distance between grid points, in which case undesired oscillations can appear. In our application, the distance from the ventricle wall to the closest region containing Doppler signal can be several $\mathrm{cm}$ as shown in Fig. 1. This problem can be solved by using a coarse grid, however this will result in fine scale flow features being missed. In this paper we propose to reconstruct velocity at multiple consecutive scales to achieve both stable and fine scale velocity reconstruction. 
Description of the Multi-scale Approach. Starting from a coarse grid (Fig. 2 (a)) with grid spacing $s=s_{0}, 3 \mathrm{D}$ velocity vectors are calculated over the whole Region of Interest (RoI), The resulting vector field is then projected along the beam direction of each view and subtracted from each input colour Doppler image, producing new images which lack coarse features calculated at that scale. These new images are used as input for the next scale where $s_{1}=s_{0} / 2$ (Fig. 2 (b)). This process is iteratively repeated for all scales (Fig. 2(c)). Our approach is inspired by a multi-level image registration framework proposed by [8], however we do not carry out simultaneous optimisation of all the scales but we deal with each scale independently at each iteration.



(a) $s=s_{0}$

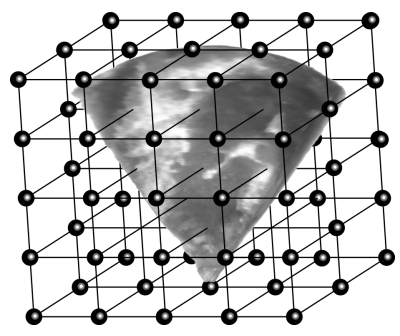

(b) $s_{1}=s_{0} / 2$.

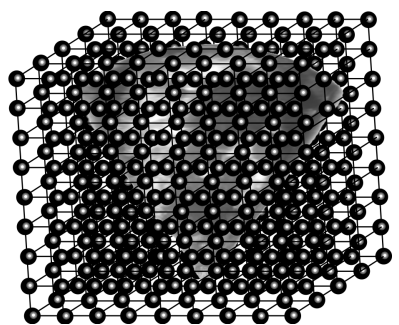

(c) $s_{2}=s_{0} / 4$.

Fig. 2. B-spline grid refinement in the multi-scale approach

\section{$3 \quad$ Experiments}

The proposed method was used to recover 3D flow using data from a 2 month old patient following aortic valve replacement for critical aortic stenosis with residual mitral stenosis. The LV had an approximate height of $43 \mathrm{~mm}$ and a diameter of $25 \mathrm{~mm}$, therefore flow reconstruction was carried out with and without wall motion and using 3 resolution scales $(s=\{12.5,6.25,3.125\}[\mathrm{mm}])$.

Flow MRI was acquired on a 1.5 Tesla Philips Achieva MR System immediately before the echo, including 2D trans-valvular flow [9] and 4D flow [MRI [10]. 2D flow MRI was used as gold standard to quantitatively validate our method.

Three colour Doppler views were consecutively acquired with a Philips X7-2 probe during breath-holds, targeting the LV chamber: 1 apical, 1 parasternal and 1 subcostal. At each view, the probe was tilted along three orientations in order to achieve maximum ventricular coverage, resulting in a total of 9 images.Doppler range was set to values from $\pm 77 \mathrm{~cm} / \mathrm{s}$ to $\pm 115.5 \mathrm{~cm} / \mathrm{s}$ depending on the view. A standard B-Mode sequence was acquired in each view for image registration. The patient was under general anaesthesia with active respiratory control. Images were acquired with institutional ethical approval after informed parental consent.

Quantitative and qualitative experiments were carried out:

- Quantitative analysis. Point-wise comparison was not possible because an accurate registration between $4 \mathrm{D}$ flow $\mathrm{MRI}$ and echo was not available. 
Instead we compared velocity distributions and inflow stroke volume (SV) calculated as the time-integral of the flow rate through the mitral valve.

- Qualitative analysis. Filling flow patterns were qualitatively compared with 4D flow MRI in terms of velocity profiles and vortex location.

\section{Results}

SV calculated with 2D flow MRI (used as reference) was $13.0 \mathrm{ml}$. The error with $4 \mathrm{D}$ flow MRI was negligible $(\mathrm{SV}=13.1 \mathrm{ml})$. SV calculated from velocity fields reconstructed from colour Doppler-derived data without wall motion information was $11.2 \mathrm{ml}$ i.e. an error of approximately $15 \%$. Using our proposed method, with wall motion information $\mathrm{SV}$ was $12.8 \mathrm{ml}$ i.e. an error with respect to $2 \mathrm{D}$ flow MRI close to $1 \%$.

Figure 3 shows the magnitude of the reconstructed velocity on three orthogonal slices (short axis, 2 chamber and 4 chamber) near the end of diastole when the ventricular vortex is fully developed. Similar planes were manually selected to capture the same location in both modalities. The first column of Fig. 3 shows velocity from $4 \mathrm{D}$ flow $\mathrm{MRI}$. The 3 rightmost columns show the velocity at three decreasing scales. At coarser scale $(s=12.5 \mathrm{~mm})$, the reconstructed velocity is almost a perfectly circular shape with smooth and homogeneous velocity distribution. At the intermediate scale $(s=6.25 \mathrm{~mm})$ flow structures are resolved at a similar resolution as $4 \mathrm{D}$ flow MRI, including a high-velocity inflow jet. At the

4D flow MRI.

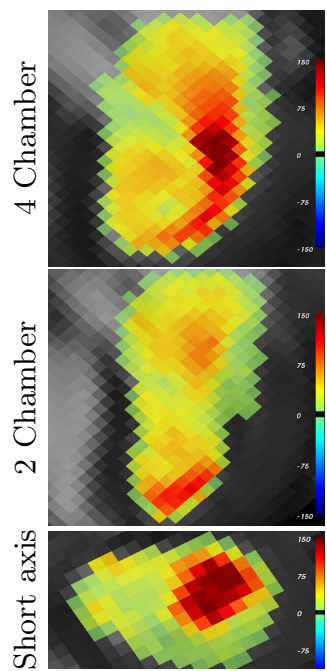

$s_{0}=12.5 \mathrm{~mm}$

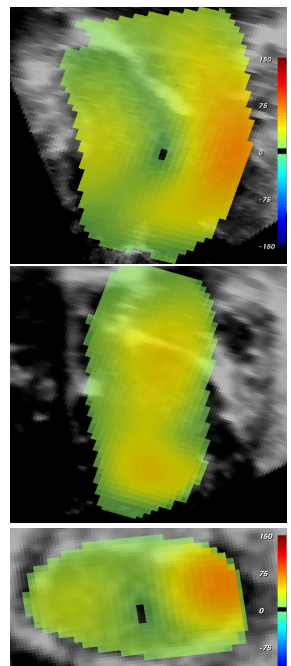

$s_{1}=6.25 \mathrm{~mm}$

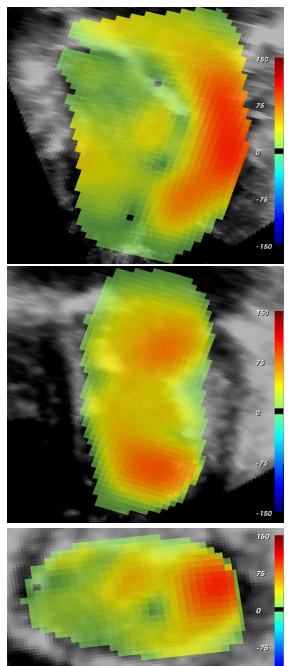

$s_{2}=3.125 \mathrm{~mm}$

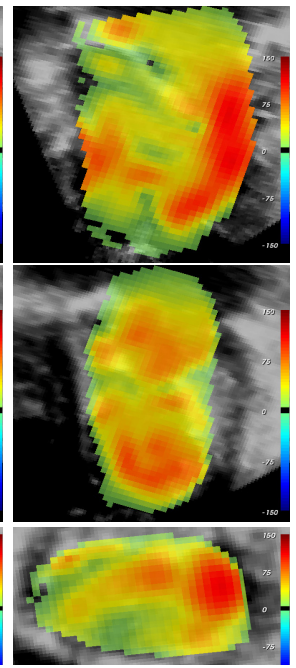

Fig. 3. Comparison between 3 cross sectional slices of the velocity magnitude, from 4D flow MRI (first column) and the proposed method at three different scales. In all cases the colour map codes velocity between -150 and $150 \mathrm{~cm} / \mathrm{s}$. 


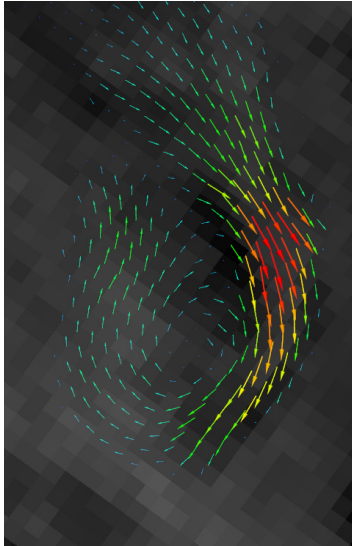

(a) 4D flow MRI.

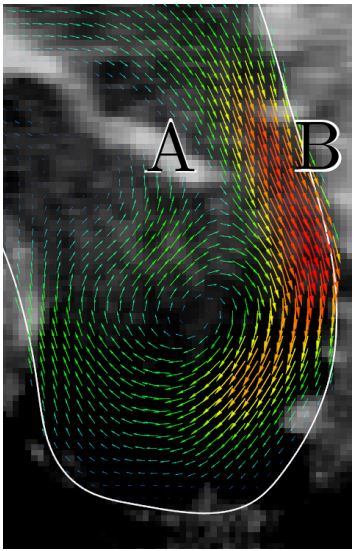

(b) Proposed method.
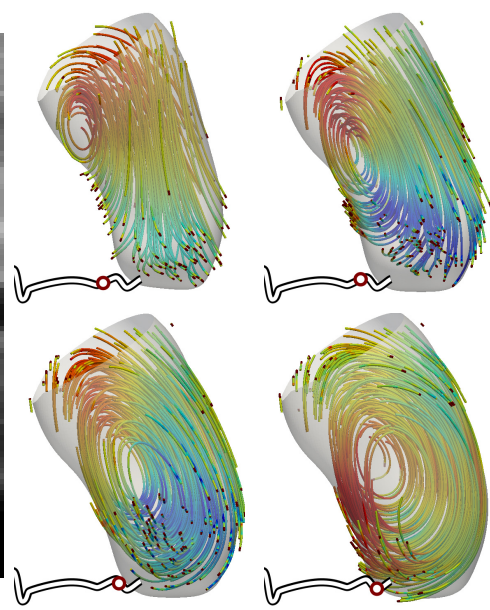

(c) Inflow vortex.

Fig. 4. Reconstructed inflow vortex. (a) $2 \mathrm{D}$ slice of $3 \mathrm{D}$ flow reconstructed from patient using $3 \mathrm{D}+\mathrm{t}$ flow MRI. Blood motion is represented by arrows which follow the velocity field from the left atrium into the LV. (b) Reconstruction, in a similar plane, with the proposed method. The limited excursion of the mitral valve leaflet 'A' produces a high velocity jet 'B'. (c) Vortex formation represented with streamlines from the 3D velocity field reconstructed with the proposed method over four diastolic times.

finest scale $(s=3.125 \mathrm{~mm})$ fine flow features emerge, some of which were not present in the MRI data. Further investigation is required to ascertain whether these fine scale structures are underlying flow features or artifacts in the reconstructed field. In what follows, we focus on the velocity reconstructed at the intermediate scale when comparing to $4 \mathrm{D}$ flow MRI.

Figure 4 shows the reconstructed flow patterns in more detail. Figure 4 (a) shows a $2 \mathrm{D}$ slice of the $3 \mathrm{D}$ velocity vectors reconstructed using $4 \mathrm{D}$ flow MRI Figure 4 (b) shows the velocity vectors reconstructed using the proposed method on a similar plane. Incorporation of wall motion allowed recovery of velocity vectors within the whole ventricle obtaining flow patterns similar to those obtained with $4 \mathrm{D}$ flow MRI. In particular the limited excursion of mitral valve leaflet ' $\mathrm{A}$ ' can be seen to create a funnelling effect causing the high velocity inflow jet ' $\mathrm{B}$ '.

In Fig. 4 (c), the formation of the intraventricular vortex is represented by streamlines at four diastolic time points. The segmentation of the ventricle is represented as a surface for anatomical reference. The streamlines represent the paths tangential to the $3 \mathrm{D}$ velocity field reconstructed with the proposed method. The increasing vortex can be clearly identified and followed through diastole. The ability to reconstruct vortices may have numerous clinical applications such as energy and efficiency analysis of the LV, and need to be further studied. 


\section{Conclusion}

We have proposed a method to recover full 3D intraventricular blood velocity from multiple 3D colour Doppler images. Specifically the method takes into account the physics of blood flow by incorporating wall motion derived from BMode images and penalising compressibility. We have demonstrated the method on one paediatric patient, where the reconstructed velocities were compared to flow MRI showing good agreement in SV, flow patterns and velocity distribution.

The present study has been carried out in a single patient and an experiment on a larger set of patients is required to assess the robustness of the method. However, this paper highlights the potential of the technique to recover complex and detailed 3D flow information with a clinically feasible protocol (data acquired in $<10 \mathrm{~min}$ ) and is particularly well-suited to small patients where flow MRI is not always possible. The wide spread availability of 3D echo systems further increases the interest and potential impact of the developed method.

\section{References}

1. de Vecchi, A., Nordsletten, D., Remme, E., Bellsham-Revell, H., Greil, G., Simpson, J., Razavi, R., Smith, N.: Inflow typology and ventricular geometry determine efficiency of filling in the hypoplastic left heart. Ann. Thorac. Surg. (2012)

2. Garcia, D., del Álamo, J.C., Tanné, D., Yotti, R., Cortina, C., Bertrand, É., Antoranz, J.C., Pérez-David, E., Rieu, R., Fernández-Avilés, F., Bermejo, J.: Two-dimensional intraventricular flow mapping by digital processing conventional color-Doppler echocardiography images. IEEE Trans. Med. Imaging 54, 1167-1178 (2010)

3. Dunmire, B., Beach, K.W., Labs, K., Plett, M., Strandness, D.E.: Cross-beam vector Doppler ultrasound for angle-independent velocity measurements. Ultrasound Med. Biol. 26(8), 1213-1235 (2000)

4. Arigovindan, M., Suhling, M., Jansen, C., Hunziker, P., Unser, M.: Full motion and flow field recovery from echo Doppler data. IEEE Trans. Med. Imaging 26(1), 31-45 (2007)

5. Gómez, A., Pushparajah, K., Simpson, J., Giese, D., Schaeffter, T., Penney, G.: A sensitivity analysis on $3 \mathrm{D}$ velocity reconstruction from multiple registered echo Doppler views. Med. Image Anal. 17(6), 616-631 (2013)

6. Grau, V., Becher, H., Noble, J.A.: Registration of multiview real-time 3-D echocardiographic sequences. IEEE Trans. Med. Imag. 26, 1154-1165 (2007)

7. Schnabel, J.A., et al.: A generic framework for non-rigid registration based on non-uniform multi-level free-form deformations. In: Niessen, W.J., Viergever, M.A. (eds.) MICCAI 2001. LNCS, vol. 2208, pp. 573-581. Springer, Heidelberg (2001)

8. Shi, W., Zhuang, X., Pizarro, L., Bai, W., Wang, H., Tung, K.-P., Edwards, P., Rueckert, D.: Registration using sparse free-form deformations. In: Ayache, N., Delingette, H., Golland, P., Mori, K. (eds.) MICCAI 2012, Part II. LNCS, vol. 7511, pp. 659-666. Springer, Heidelberg (2012)

9. Giese, D., Schaeffter, T., Kozerke, S.: Highly undersampled phase-contrast flow measurements using compartment-based K-t principal component analysis. Magnet. Reson. Med. 69(2), 434-443 (2012)

10. Giese, D., Greil, G., Schaeffter, T., Kozerke, S.: Highly accelerated 4D MR flow measurements in congenital heart disease. In: ISMRM 2012, Number 0665 (2012) 